\title{
Vision of conspecifics decreases the effectiveness of ethanol on zebrafish behaviour
}

\author{
Rachel Dean ${ }^{1}$, Nicole Hurst-Radke ${ }^{1}$, Nirudika Velupillai ${ }^{2}$, Brian C Franczak ${ }^{2}$, Trevor J Hamilton ${ }^{\text {Corresp. } 1,3}$ \\ 1 Department of Psychology, MacEwan University, Edmonton, Alberta, Canada \\ 2 Department of Mathematics and Statistics, MacEwan University, Edmonton, Alberta, Canada \\ 3 Neuroscience and Mental Health Institute, University of Alberta, Edmonton, Alberta, Canada \\ Corresponding Author: Trevor J Hamilton \\ Email address: trevorjameshamilton@gmail.com
}

Aquatic organisms in pharmacology and toxicology research are often exposed to compounds in isolation prior to physiological or behavioural testing. Recent evidence suggests that the presence of conspecifics during a stressful event can modulate behavioural outcomes (called 'social buffering') when testing occurs within the same context. It is unknown, however, whether the social environment during exposure interacts with the efficacy of anxiety-altering substances when subsequently tested in the absence of conspecifics. In this study, zebrafish were individually exposed to habitat water or ethanol $(1.0 \% \mathrm{vol} / \mathrm{vol})$ while untreated conspecifics were visually present or absent during dosing. Using the novel object approach test, a validated test of boldness and anxiety-like behaviour, we observed significantly greater effects of ethanol in isolated fish, compared to fish with a view of conspecifics during dosing. These results were not explained by altered locomotion during exposure, which might otherwise increase drug uptake. This highlights the need to consider the social environment during exposure when conducting and interpreting behavioural research involving drug or toxicant exposure. 
${ }^{1}$ Department of Psychology, MacEwan University, Edmonton, AB, Canada.

${ }^{2}$ Department of Mathematics and Statistics, MacEwan University, Edmonton, AB, Canada.

11

12 13

Corresponding Author: Trevor James Hamilton

${ }^{3}$ Neuroscience and Mental Health Institute, University of Alberta, Edmonton, AB, Canada.

\section{Email address: trevorjameshamilton@gmail.com}


39 Aquatic organisms in pharmacology and toxicology research are often exposed to compounds in

40 isolation prior to physiological or behavioural testing. Recent evidence suggests that the presence

41 of conspecifics during a stressful event can modulate behavioural outcomes (called 'social

42 buffering') when testing occurs within the same context. It is unknown, however, whether the

43 social environment during exposure interacts with the efficacy of anxiety-altering substances

44 when subsequently tested in the absence of conspecifics. In this study, zebrafish were

45 individually exposed to habitat water or ethanol $(1.0 \% \mathrm{vol} / \mathrm{vol})$ while untreated conspecifics

46 were visually present or absent during dosing. Using the novel object approach test, a validated

47 test of boldness and anxiety-like behaviour, we observed significantly greater effects of ethanol

48 in isolated fish, compared to fish with a view of conspecifics during dosing. These results were

49 not explained by altered locomotion during exposure which might otherwise increase drug

50 uptake. This highlights the need to consider the social environment during exposure when

51 conducting and interpreting behavioural research involving drug or toxicant exposure. 
Living in a social environment offers many evolutionary advantages. Belonging to a

60

61

62

63

64

group can facilitate reproduction, enable earlier detection and evasion of predators, and improve resource efficiency (Rubinstein, 1978). Social cues are commonly the mechanism that conveys these messages between conspecifics and can guide responses in uncertain situations (Suboski et al., 1990). Another benefit of conspecifics is a decrease in stress level that minimizes the impact of stressful situations (Kikusui, Winslow \& Mori, 2006). This phenomenon, known as 'social buffering', has been experimentally demonstrated in many species including cats (Masserman, 1943), goats (Liddell, 1949), rats (Davitz \& Donald, 1955; Latané, 1969), humans (Hostinar, Johnson \& Gunnar, 2015), and, recently, zebrafish (Oliveira \& Faustino, 2017; Faustino, TacãoMonteiro \& Oliveira, 2017).

The zebrafish has become a popular model organism for use in a variety of scientific disciplines including pharmacology and toxicology. Behavioural neuroscience tests can be used to analyze a wide variety of cognitive processes in zebrafish including episodic-like memory (Hamilton et al., 2016), object recognition memory (May et al., 2016), classically conditioned memory (Sison \& Gerlai, 2010), fear (Speedie \& Gerlai, 2008), boldness (Dean et al., 2020), and anxiety-like behaviour (Maximino, de Brito \& da Silva Batista, 2010). To test anxiety-like behaviour there are a variety of paradigms available, with the most common being the light/dark preference and novel tank diving tests (for a review see (Maximino, de Brito \& da Silva Batista, 2010). Due to the reliability of these tests and the practical simplicity in which psychopharmacological substances can be administered to zebrafish (Gerald, Lee \& Blaser, 2006), adaptive behavioural responses can be easily manipulated with anxiolytic (anxietyreducing) and anxiogenic (anxiety-enhancing) compounds (Collier \& Echevarria, 2013). 

anxiety-like behaviour in a variety of paradigms. For instance, $0.25 \%, 0.5 \%$ and $1.0 \%$ ethanol increase the amount of time zebrafish spend exploring the light zone of the light/dark test

84 (Gebauer et al., 2011; Fontana et al., 2020) and $0.3 \%$ and $0.5 \%$ ethanol increase time spent in the top zone of a novel tank dive test (Egan et al., 2009; Müller et al., 2020), both indications of the anxiolytic nature of ethanol. In the novel object approach test, used to test boldness and anxietylike behaviour, $1.0 \%$ ethanol reduces the amount of time zebrafish spend in the outer 'thigmotaxis zone' adjacent to the wall of the arena (Johnson \& Hamilton, 2017) further exemplifying the anxiolytic nature of ethanol. Using the same test, $1.5 \%$ ethanol also increases the time zebrafish spend in the inner zone exploring a novel object (Hamilton et al., 2017b), thus increasing boldness. Taken together, these findings illustrate zebrafish display less anxiety and more boldness following an acute administration of ethanol. Recent evidence, however, suggests that the social environment in which anxiety-altering compounds are administered and/or tested in may influence the behavioural effects of these substances in zebrafish (Faustiona, TacãoMonteiro \& Oliveira, 2017), which may complicate conclusions. anxiogenic compound when exposure and testing occurs within the same environment (Faustino, Tacão-Monteiro \& Oliveira, 2017). Specifically, conspecific water and alarm substance along with a visual of untreated conspecifics induced significantly less freezing and erratic movements than when the adjacent tank was empty and no conspecific water was added (Faustino, Tacão-

101 Monteiro \& Oliveira, 2017). When the effectiveness of each type of cue was tested, visual cues

102 were superior to olfactory cues in reducing aversive behaviours and promoting 'social buffering'

103 (Faustino, Tacão-Monteiro \& Oliveira, 2017). However, it is unknown whether the presence of 
104 conspecifics can alter behavioural response when the fish are subsequently removed from the

105 compound and tested in a separate arena. In other words, does the effect of social buffering

106 persist beyond the exposure to the cues?

107 In the majority of acute pharmacological experiments that test individual fish behaviour,

108 substances are administered while fish are physically isolated, and the exposed fish is then

109 transferred to a behavioural arena for testing (Stewart et al., 2012). Moreover, almost all of the

110 studies published that have examined acute ethanol exposure on behaviour of individual fish

111 have not specified whether conspecifics were within view during dosing (Blaser and Peñalosa,

112 2011, Echevarria et al., 2008; Egan et al., 2009, Fontana et al., 2020, Gerlai et al., 2008).

113 Moreover, only a few other studies have stated that fish were isolated during ethanol dosing

114 (Hamilton et al., 2017b, Johnson and Hamilton 2010). To the best of our knowledge, no study

115 has examined whether the view of conspecifics during dosing may influence the anxiety level of

116 a fish subsequently tested in an isolated testing arena. It is also unknown whether social

117 buffering may also act to alter the effects of anxiety reducing, 'anxiolytic' substances. To test

118 these questions, we exposed individual zebrafish to either habitat water or ethanol (1.0\% vol/vol)

119 while untreated conspecifics were visually present or absent for the entire exposure period.

120 Following exposure, the fish were transferred to the novel object approach test for quantification

121 of anxiety-like behaviour and boldness (Dean et al., 2020; Krook et al., 2019; Leighton et al.,

122 2018). Finally, we tested whether fish move at different rates and remain closer to conspecifics

123 during the dosing period itself, in order to determine whether the social condition (Isolated vs.

124 In-view) influences behaviour during drug exposure.

125

126 
127

128

129

130

131

132

133

134

135

136

137 animals.

143 Isolated-CTL, Isolated-Ethanol, In-view-CTL, and In-view-Ethanol. Following exposure,

150 behaviour was tested in the novel object approach test (Fig. 1C,D) to examine whether the social 151

\section{Subjects and housing}

\section{METHODS}

Short-fin wild-type zebrafish $(\mathrm{n}=90)$ were acquired from Aquatic Imports (Calgary, AB) at a minimum age of 9-months. Fish were experimentally naïve and comprised of mixed males and females ( $\sim 50 / 50$ ratio). Following a month-long quarantine period, the fish were held in either 3 or 10L polypropylene tanks within a three-shelf bench top system (Aquatic Habitats, Aquatic Ecosystems, Inc. Apopka, FL, USA) which was controlled for filtration and aeration. No fish was ever housed in isolation and tank capacities never exceeded five fish per liter. Temperature and $\mathrm{pH}$ remained between $26-30^{\circ} \mathrm{C}$ and $6.0-8.0$, respectively. Lights were kept on a 12-hour light/dark cycle with lights on at 8AM and off at 8PM. Fish were fed dry brine shrimp (Omega One Freeze Dried Mysis Shrimp nutri-treat, OmegaSea Ltd., Germany) once per day, and after experimentation on test days. All experiments were approved by the MacEwan University Animal Research Ethics Board (AREB) under protocol number 05-12-13 in compliance with the Canadian Council for Animal Care (CCAC) guidelines for the care and use of experimental animals.

\section{Experimental design}

Experiment 1: This experiment used a $2 \times 2$ factorial design. The between-subject experimental variables included visual access to conspecifics (Fig. 1A,B, Isolated or In-view) and the type of substance the fish were exposed to (habitat water $(C T L)$ or Ethanol) while in the dosing containers. Prior to experimentation, fish were randomly assigned to one of four groups: environment during exposure influences the efficacy of ethanol. 
152 Experiment 2: A follow-up experiment was performed in which we tested the movement of

153 individual zebrafish within the dosing containers in the Isolated or In-view conditions while

154 exposed to control water (Fig. 2A,B).

155

\section{Experiment 1: Experimental conditions}

157 Ethanol exposure: On the day of experimentation, fish were carried in their habitat tanks into the experimental room prior to feeding and were given at least 10 minutes to acclimatize to this new environment. A white corrugated plastic barrier was set up surrounding habitat tanks to limit external stimuli. Following the habituation period, fish were individually netted from their habitat tanks and placed into one of two experimental dosing containers $(600 \mathrm{~mL})$. Each dosing container contained $500 \mathrm{~mL}$ of solution and was also surrounded by white corrugated plastic

164 by allowing two fish to be dosed with a staggered schedule. Once in the dosing container, a square piece of the same plastic was placed on top to prevent evaporation of the solution and to ensure fish remained inside (Cachat et al., 2010; Holcombe et al., 2013). Fish exposed to control water (Isolated-CTL $(\mathrm{n}=15)$ or In-view-CTL $(\mathrm{n}=15))$, were placed into dosing containers that only contained habitat water $(500 \mathrm{~mL})$. Fish in the ethanol groups (Isolated-Ethanol $(\mathrm{n}=15)$ or In-view-Ethanol $(\mathrm{n}=15))$ were placed into dosing containers with $1.0 \%$ ethanol. Solutions for each compound were made fresh each day by mixing $5.26 \mathrm{~mL}$ of non-denatured, $95 \%$ ethanol

171 into $495 \mathrm{~mL}$ of habitat water in the respective dosing containers. The selected concentration and

172 duration of ethanol exposure was based on previous experiments in zebrafish (Johnson \&

173 Hamilton, 2017). 
174 View of conspecifics: Fish were assigned to one of the two Isolated conditions (Isolated-CTL (n

$175=15)$, or Isolated-Ethanol $(\mathrm{n}=15))$ with no view of conspecifics while in the dosing container,

176 or fish were assigned to the In-view conditions (In-view-CTL $(\mathrm{n}=15)$ or In-view-Ethanol $(\mathrm{n}$

$177=15)$ ), with a view of a tank containing 12 untreated conspecifics during dosing. The dosing

178 containers used in the In-view conditions were positioned in front of each other to ensure fish in

179 both dosing containers had equal view of their conspecifics. The same group of conspecifics

180 were used for each In-view condition. A white corrugated plastic barrier covered the remaining

181 two sides of the conspecific tank (Fig. 1B) and water temperatures were maintained between 26

182 and $30^{\circ} \mathrm{C}$ by seedling heat mats (Hydrofarm Horticultural Products, Petaluma CA). Fish in the

183 Isolated condition were surrounded fully by a white plastic barrier which was also placed on the

184 heat mat. Fish in all conditions remained in the dosing containers for 30 minutes, after which the

185 solution (including the fish) was carefully poured into a net, with a second dosing container

186 collecting the solution. Once in the net, the fish was placed into the adjacent behavioural arena

187 for testing. No data was acquired during dosing in experiment 1.

188

\section{Experiment 1: Behavioural testing}

190 Fish were individually tested in the novel object approach test following the 30-minute exposure

191 period. The behavioural arena used in this experiment was circular and made from white opaque

192 plastic $(\varnothing=34 \mathrm{~cm}$; depth $=15 \mathrm{~cm}$; Fig. $1 \mathrm{C})$. The arena was placed on top of a heat mat to

193 maintain habitat water temperatures and was surrounded by a three-sided white corrugated

194 plastic enclosure to limit external stimuli during testing. Habitat water was added to the arena up

195 to a maximum height of $5 \mathrm{~cm}$ and was replaced with fresh habitat water every four hours. An

196 equal amount of heated habitat water was also exchanged whenever temperatures fell below 
$19726^{\circ} \mathrm{C}$. The object used in this study was a $2 \mathrm{~cm}$ x $4.25 \mathrm{~cm}$ Lego figurine which was multi-

198 coloured to rule out possible colour preferences (Fig. 1D; Dean et al., 2020; Hamilton et al.,

199 2017b; Johnson \& Hamilton, 2017) and was adhered using velcro to the bottom of the arena's

200 center. Prior to testing, three virtual zones representing the thigmotaxis (the outermost zone, 23-

$20134 \mathrm{~cm}$ in diameter), inner (center zone of $12 \mathrm{~cm}$ in diameter) and transition sones (in between the

202 other two zones, $12-23 \mathrm{~cm}$ in diameter) were defined using EthoVision XT motion tracking

203 software (Fig. 1E; version 11.0, Noldus, VA, USA). All experimental procedures occurred

204 between 9AM and 6PM prior to feeding. The time, in seconds, fish spent in each zone

205 (thigmotaxis, transition, inner) was recorded and used as a proxy for anxiety-like behaviour (i.e.

206 increased time in the thigmotaxis zone related to increased anxiety) and boldness behaviour (i.e.

207 increased time in the inner zone is related to increased boldness) (Ou et al., 2015). Locomotion

208 was also assessed by tracking the distance $\operatorname{moved}(\mathrm{cm})$ and immobility(s). Fish were tested

209 individually for a period of 10 minutes following dosing and recording began as soon as the fish

210 was placed into the transition zone facing the object.

211

\section{Experiment 2: Experimental conditions and behavioural testing}

213 After the first round of experimentation we sought to determine if the social context during

214 exposure affected the distance fish moved while in the dosing container; possibly this could

215 account for any differences in behavioural outcomes observed? In a second experiment, we

216 tested the activity of a new group of fish in the dosing containers while in either the Isolated-

$217 C T L(\mathrm{n}=15)$ or In-view-CTL $(\mathrm{n}=15)$ condition. We also quantified whether fish preferred the half

218 of the dosing container close to conspecifics (conspecific side). Following a 10-minute

219 habituation period, one fish was individually netted from their habitat tank and placed into a 600 
$220 \mathrm{~mL}$ dosing container with habitat water $(500 \mathrm{~mL})$, identical to procedures in our first experiment.

221 A rectangular piece of white corrugated plastic was placed beneath the dosing container to assist

222 with motion tracking. As in the novel object approach test, a three-sided enclosure was set up

223 during behavioural tracking and seedling heat mats maintained water temperatures. To ensure

224 these fish received the same treatment as fish in the Isolated-CTL and In-view-CTL conditions in

225 the first experiment, a white piece of corrugated plastic was also set up across the front of the

226 three-sided enclosure (Fig. 2A). For fish in both the Isolated-Dosing and In-view-Dosing

227 conditions, EthoVision was set up to record the distance $(\mathrm{cm})$ each fish moved throughout the

228 30-minute exposure period while in the dosing container. For fish in the In-view-Dosing

229 condition, a habitat tank containing the same conspecifics $(\mathrm{n}=12)$ that were used in the other In-

230 view conditions was positioned to the right of the beaker. Using EthoVision, the beaker was then

231 vertically split into two equal-sized virtual sections to compare the amount of time, in seconds,

232 fish explored the side of the beaker closest to conspecifics (conspecific side) and the side farthest

233 from conspecifics (empty side; Fig.2B). To rule out external variables potentially contributing to

234 a side preference, the habitat tank was placed to the left of the beaker for the final three of fifteen

235 trials per condition. No differences were observed in the time spent exploring either side of the

236 beaker regardless of whether the habitat tank was on the right or left side of the beaker (Mann-

237 Whitney; conspecific side, $\mathrm{U}=10, \mathrm{p}=0.2549$; opposite side, $\mathrm{U}=10, \mathrm{p}=0.2945$ ) so these were 238 combined for analysis.

\section{Statistical analysis}

$241 \mathrm{R}$ (version 4.0.2) was used to analyze the data. The majority of the utilized functions are a part of

242 the stats library. The exceptions are the brunnermunzel.test and leveneTest functions, which are a 
243 part of the brunnermunzel and car libraries, respectively (Fox, 2016; Fox and Weisberg, 2019;

244 Munzel and Brunner, 2000). Prior to the model fitting process, an exploratory data analysis was

245 performed on all variables. In particular, we used the cor.test function to determine if there was

246 evidence of statistically significant correlations among the measurements in the thigmotaxis,

247 transitions, and inner zones and among the measurements in the distance moved and immobility

248 variables. To analyze the effect of social condition and/or ethanol on anxiety levels, two-way

249 ANOVAs were fitted to each variable using the aov function. The normality and constant

250 variance assumptions were checked using Shapiro-Wilks' and Levene's tests, respectively, via

251 the shapiro.test and leveneTest functions. If either assumption was violated, a Gamma

252 Generalized Linear Model (GLM) with log link was fitted to the data using the glm function. If

253 there was evidence that the Gamma GLM did not sufficiently fit the data, then Wilcoxon rank-

254 sum (WRS) tests were fitted to compare both main effects and the treatment combinations of

255 interest via the wilcox.test and pairwise.wilcox.test functions. In cases where the Gamma GLM

256 fit sufficiently, the Brunner-Munzel test (Munzel and Brunner, 2000) was used to analyze

257 differences between the treatment combinations of interest via the brunnermunzel.test function.

258 Significance across all tests was determined using a 5\% significance level. In what follows, we

259 use $\mathrm{t}(\mathrm{df})$ to designate a Student's $t$ test statistic with df degrees of freedom, $\mathrm{F}(\mathrm{df} 1, \mathrm{df} 2)$ to

260 represent a F-test statistic with df1 and df2 degrees of freedom, $\mathrm{W}$ to represent the test statistic

261 from a Wilcoxon Rank Sum test, and $\mathrm{W}_{\mathrm{BM}}$ to represent the test statistic from the Brunner-

262 Munzel test.

263

264

RESULTS

\section{Analysis of Correlation}


266 Time spent in the thigmotaxis zone was negatively correlated with time spent in the transition

267 zone $(\mathrm{r}=-0.816, \mathrm{t}(58)=-10.74, \mathrm{p}<0.001)$ and time spent in the transition zone $(\mathrm{r}=-0.754, \mathrm{t}(58)$

$268=-8.74, \mathrm{p}<0.001)$. On the contrary, time spent in the inner zone was positively correlated, but

269 the result was not statistically significant $(\mathrm{r}=0.235, \mathrm{t}(58)=1.84, \mathrm{p}=0.071)$. Distanced moved

270 was significantly negatively correlated with time spent immobile $(r=-0.643, t(58)=-6.400, p<$

271 0.001). While the results show that there are significant correlations between the times spent in

272 each zone and between distance moved and immobility we follow Hamilton et al., (2017) and

273 Thomson et al., (2020) and study individual behavioural measures rather than consider a

274 principal component analysis as it has been shown that these behavioural measures have a large

275 variance across reports from different research groups.

276

277 Effect of social context

278 Social context was found to have a significant effect on behaviour when comparing the time fish

279 in the In-view and Isolate conditions spent in the thigmotaxis, transition, and inner zones.

280 Specifically, fish spent more time in the thigmotaxis zone in the In-view condition than they did

281 in the Isolate condition $(t(56)=4.26, p<0.0001$; Fig. $3 \mathrm{~A})$, and less time in the transition zone

$282(t(56)=3.98, p<0.001$; Fig. 3B $)$ and the inner zone $(t(56)=3.40, p=0.001$; Fig. 3C $)$, on

283 average. No significant differences were found between the mean distance fish in the In-view and

284 Isolate conditions moved $(F(1,56)=2.44, p=0.12$; Fig. 3D) nor between the median time fish in

285 these groups spent immobile ( $W=470, p=0.77$; Fig. $3 \mathrm{E})$.

286

287 Investigating social context interactions 
Among the control (CTL) groups, the Brunner-Munzel test provided sufficient evidence

289

290

291

292

293

294

295

296

297

298

299

300

301

302

303

304

to conclude that fish in the Isolate-CTL group spent more time in the thigmotaxis zone than fish in the In-View-CTL group $\left(W_{B M}=-4.34, p<0.001\right.$, Fig. 3A). Whereas, fish in the In-View-CTL group spent more time in both the inner and transition zones than fish in the Isolate-CTL group $\left(W_{B M}=3.99, p<0.001\right.$, Fig. $3 \mathrm{C}$ and $W_{B M}=4.92, p<0.0001$, Fig. 3B respectively).

Among the ethanol groups, the Brunner-Munzel test provided sufficient evidence to conclude that fish in the In-view-Ethanol group spent more time in the thigmotaxis zone than fish in the Isolate-Ethanol group $\left(W_{B M}=2.07, p=0.05\right.$, Fig. $\left.3 \mathrm{~A}\right)$. Whereas, fish in the IsolateEthanol group spent more time in the inner zone compared to fish in the In-View-Ethanol group $\left(W_{B M}=-2.34, p=0.03\right.$, Fig. $\left.3 \mathrm{C}\right)$. No differences were found when comparing the time fish in the Isolate-Ethanol and In-View-Ethanol groups spent in the transition zone ( $W_{B M}=-0.93, p=0.361$, Fig. 3B).

\section{Effect of type of solution}

The type of solution was found to have a significant effect on fish behaviour when comparing the time fish spent in their habitat water (CTL) to the time fish spent in ethanol. In particular, we

found that fish dosed in the ethanol solution spent less time in the in the thigmotaxis zone $(\mathrm{t}(56)$ $=8.78, p<0.0001$; Fig. $3 \mathrm{~A})$, and more time in the transition zone $(\mathrm{t}(56)=7.20, p<0.0001$; Fig.

$3 \mathrm{~B})$ and the inner zone $(\mathrm{t}(56)=9.31, p<0.0001$; Fig. 3C), on average, compared to fish dosed in control water. In addition, a significant difference was also detected between the median time spent immobile when comparing fish in their habitat water to those in ethanol $(W=169.5, p<$ 0.0001). No significant difference was found when comparing the mean distance moved for 
310 these two groups $(F(1,56)=3.75, p=0.058$; Fig. 3D).

311

\section{Investigating solution interactions}

313

314

315

316

317

318

319

320

321

322

323

324

325

326

327

328

329

330

331

Investigating the treatment combinations revealed that time spent in the thigmotaxis zone was statistically greater for fish in the In-view-CTL group compared to fish in the In-view-

Ethanol group $\left(W_{B M}=-3.24, p=0.003\right.$, Fig. 3A). Whereas, time spent in the transition zone was statistically greater for fish in the In-view-Ethanol group compared to fish in the In-view-CTL group $\left(W_{B M}=3.52, p=0.002\right.$, Fig. 3B). A significant difference was not found when comparing the time fish in these two groups spent in the inner zone $\left(W_{B M}=1.82, p=0.079\right.$, Fig. 3C). In addition, the Wilcoxon rank-sum test concluded that the median time spent immobile for fish in the In-view-Ethanol group was not significantly different compared to fish in In-view-CTL group $(\mathrm{W}=69, \mathrm{p}=0.074$, Fig. 3E).

Post hoc comparison of the isolated treatment groups using the Brunner-Munzel test indicated that fish in the Isolate-CTL group spent more time in the thigmotaxis zone than fish in the Isolate-Ethanol group $\left(W_{B M}=-32.19, p<0.0001\right.$, Fig. 3A). Whereas, fish in the IsolateEthanol group spent more time in both the transition zone $\left(W_{B M}=24.80, p<0.0001\right.$, Fig. 3B) and the inner zone $\left(W_{B M}=13.16, p<0.0001\right.$, Fig. 3C) compared to fish in Isolate-CTL group. The Wilcoxon rank-sum test indicated that the median time spent immobile by fish in IsolateCTL group was significantly greater than median time spent in the in Isolate-Ethanol group $(\mathrm{W}=$ 16.5, $\mathrm{p}<0.001$, Fig. 3E). 
333 between Isolated-Dosing and In-view-Dosing groups $\left(\mathrm{t}_{28}=1.255, p=0.2198 ;\right.$ Fig. 4A $)$. A highly

334 significant preference for the conspecific side of the dosing container was found in fish from the

335 In-view-Dosing group $\left(\mathrm{t}_{28}=10.21, p<0.0001\right.$; Fig. 4B).

\section{DISCUSSION}

338 To examine whether the sight of conspecifics during dosing impacts effectiveness of anxiety-like

339 behaviour measurements and anxiety-altering substances, we exposed zebrafish to habitat water

340 or ethanol $(1.0 \%)$ while fish were isolated or able to observe conspecifics. Following dosing,

341 behaviour was tested in the novel object approach test. Fish that were able to view conspecifics

342 during dosing with control water had significantly less anxiety-like behaviour and increased

343 boldness compared to fish dosed in isolation. The behavioural effects of ethanol also varied

344 depending on the social condition in which it was administered. Ethanol had a significantly

345 greater effect on anxiety-like behaviour and boldness in isolated fish compared to fish that were

346 able to view conspecifics during dosing.

347 Both the sight of conspecifics and ethanol exposure significantly increased the time fish

348 spent in the zones closest to the novel object (transition and inner; Fig. 3B-C), consistent with

349 previous research demonstrating that ethanol increases boldness (Hamilton et al., 2017b; Johnson

$350 \&$ Hamilton, 2017). Ethanol and sight of conspecifics also both decreased time spent in the

351 thigmotaxis zone; an indication that anxiety-like behaviour was decreased. The most pronounced

352 change in behaviour occurred with the combination of ethanol exposure and isolation during

353 dosing compared to ethanol exposure and view of conspecifics; anxiety-like behaviour was

354 significantly reduced (Fig. $3 \mathrm{~A}, \mathrm{P}<0.0001$ ) and boldness was increased (Fig. $3 \mathrm{C}, \mathrm{P}<0.0001$ ). In 
355 fish with a view of conspecifics, ethanol still did decrease anxiety-like behaviour, however to

356 much less of an extent than in isolated fish, and had little effect on approach to the novel object.

357 A similar pattern emerged in ethanol's influence on locomotion. Ethanol did not impact the

358 distance Isolated or In-view fish moved (Fig. 3D), or the time In-view fish spent immobile; it

359 only increased immobility in Isolated fish (Fig. 3E). This suggests that social isolation either

360 increases sensitivity to ethanol's anxiolytic and depressant effects, or the presence of

361 conspecifics suppresses these effects.

362 In an attempt to understand how the social context contributes to differences observed in

363 behavioural outcomes, we analyzed the behaviour of a second group of fish while in the dosing

364 container during the 30-minute dosing period. Because mobility may affect the rate of intake

365 when fish are dosed via immersion, with greater physiological demands resulting in more

366 ventilation and therefore increased uptake of the drug through the gills (Blaser \& Vira, 2014), we

367 sought to determine whether the heightened effect of ethanol observed in Isolated fish could be

368 explained by greater movement during dosing. We analyzed the behaviours of fish exposed to

369 habitat water while isolated or within view of conspecifics while they were in the dosing

370 container. Interestingly, no differences were observed in the distance fish moved (Fig. 4A),

371 indicating differences in locomotion during dosing could not explain the behaviours we

372 observed. Not surprisingly, zebrafish spent significantly more time on the side of the dosing

373 container closest to conspecifics when in view (Fig. 4B), demonstrating their preference to

374 remain near other zebrafish.

375 An explanation for the increased anxiolytic effect of ethanol in Isolated relative to In-

376 view groups may be related to 'social buffering.' Previous research in zebrafish has shown that

377 the presence of conspecifics helps to suppress anxiety evoked by a fearful stimulus (Faustino, 
378 Tacão-Monteiro \& Oliveira, 2017). Faustino, Tacão-Monteiro and Oliveira (2017) first

379 demonstrated this in zebrafish by exposing fish to a conspecific alarm substance with or without

380 the presence of conspecific cues. They found that the anxiogenic effects were dampened by the

381 presence of olfactory and/or visual cues. In other words, fish that could observe or smell their

382 conspecifics showed less anxiety in response to the alarm substance (Faustino, Tacão-Monteiro

383 \& Oliveira, 2017). The mechanisms of social buffering have not been well explored in zebrafish;

384 however, it is possible that zebrafish use the behaviours of their conspecifics as a source of

385 information to guide their own responses in unfamiliar or fearful environments. This would

386 explain why there was less of an effect of ethanol in the In-view condition in our experiment (ie.

387 higher time in the thigmotaxis zone and less time near the object) compared to the Isolated

388 condition. Presumably, fish in the dosing container were observing their conspecifics behaving

389 normally and this may have minimized the effect of ethanol. However, social buffering has only

390 been shown to decrease stress responses and in our study anxiolysis was reduced. The effect of

391 ethanol may have been 'buffered' by the presence of conspecifics. The sight of conspecifics did

392 significantly lower time spent in the thigmotaxis zone independent of the presence of ethanol,

393 which suggests that the sight of conspecifics may lower anxiety overall. However, if this were

394 the only factor at play then the effect of ethanol should be greater in the In-view condition

395 compared to the Isolated condition, because of the combined anxiolytic effect of conspecifics

396 and the anxiolytic effect of ethanol, but this is the opposite of what we observed. Somehow the

397 visual cues from conspecifics decreased the effectiveness of ethanol, and the candidate

398 mechanism is social mimicry, which has been demonstrated in zebrafish (Dresoti et al., 2015).

399 To further investigate this future, studies could manipulate the emotional state of conspecifics

400 with stress or pharmacology then examine how these In-view fish affect the behavioural 
401 outcomes of the individual fish observing them. In this study, we did not examine sex differences

402 and we did not manipulate the number of fish used as conspecifics nor the distance of the

403 conspecific tank from the focal fish. These factors may also impact the strength of the social

404 buffering response. It would also be valuable to explore the neurochemical basis of social

405 buffering with analysis of brain chemistry after dosing in these social conditions.

406

407

CONCLUSIONS

408 Taken together, the sight of conspecifics decreases anxiety, and more so, buffers the anxiolytic

409 effect of ethanol. These findings have important implications in the fields of pharmacology,

410 toxicology and behavioural neuroscience as isolated drug administration seems to be more

411 effective in eliciting a behavioural response. Additionally, zebrafish behavioural research

412 findings can be inconsistent across laboratories, and this may be due a lack of detailed

413 methodological reporting, including whether conspecifics are within visual range during drug

414 exposure. Social buffering may offer a potential explanation for at least some of these

415 discrepancies and necessitates detailed descriptions of dosing conditions in future experiments.

416 Overall, this study provides the first evidence that the social condition during dosing effects the

417 efficacy of anxiolytic substances when subsequently tested in isolation. This highlights the need

418 to consider the social environment during exposure when conducting or interpreting behavioural

419 research.

420 
421 Figure legends:

422 FIGURE 1. Experimental dosing set-up. (A) Isolated and (B) In-view dosing. During dosing an

423 individual fish was netted from the holding tank and placed into one of the two dosing

424 containers. In-view fish had visual access to 12 conspecifics held in the conspecific tank but were

425 not able to see the other fish being dosed. Focal fish remained in the dosing containers for 30-

426 minutes prior to behavioural testing. C) The circular arena used was $34 \mathrm{~cm}$ in diameter and 16

$427 \mathrm{~cm}$ in height. D) The novel object used was a multi-coloured LEGO® figurine. E) The

428 thigmotaxis, transition and inner zones were calibrated to 34,23 and $12 \mathrm{~cm}$ in diameter

429 respectively.

430

431 FIGURE 2. Experimental set-up for motion-tracking during exposure. (A) Isolated dosing, or

432 (B) In-view dosing. The circle in the bottom left of figure (B) represents the virtual zones created

433 in EthoVision to test whether fish spend more time on the side of the beaker closest to

434 conspecifics when in view.

435

436 FIGURE 3. Effects of social context and ethanol on zone preference. The time, in seconds, fish

437 spent in the thigmotaxis (A), transition (B) and inner zones (C). (D) and (E) represent the effect

438 of social context and substance on the distance fish moved (D) and the time fish spent immobile

439 (E). Graphs show individual data points ( $n=15$ per group) with horizontal lines that represent

440 mean values \pm SEM. The number of * symbols identifies significant differences between groups

441 at varying levels of significance: $* P<0.05 ; * * P<0.01 ; * * * P<0.001 ; * * * * P<0.0001$.

442 
443 FIGURE 4. Distance and side preferences during the dosing procedure. (A) The distance,

444 Isolated-CTL and In-view-CTL fish moved and the (B) amount of time, in seconds, In-view-

445 CTLs spent on either side of the dosing container during dosing. The social condition did not

446 have a significant effect on the distance fish moved, however when in-view, fish had a

447 significant preference for the side of the dosing container closest to conspecifics. Data was

448 analyzed using independent $t$-tests. Graphs show individual data points $(\mathrm{n}=15$ per group)

449 with horizontal lines that represent mean values \pm SEM. The number of * symbols identifies

450 significant differences between groups at varying levels of significance: $* * * * P 0.0001$.

\section{Acknowledgements}

452 We would like to thank Jasmin Bajwa, Shayna Chaput, Dr. Melike Schalomon, and Aleah

453 McCory (Animal Care Technician) for their help with daily husbandry and aquarium

454 maintenance.

455

456

457

458

459 
460

461

462

463

464

465

466

467

468

469

470

471

472

473

474

475

476

477

478

479

480

481

482

483

484

485

486

487

488

489

490

491

492

493

494

495

496

497

498

499

500

501

502

503

504

\section{References}

Blaser RE, Pénalosa YM. 2011. Stimuli affecting zebrafish (Danio rerio) behavior in the light/dark preference test. Physiology \& Behavior, 104: 831-838. DOI:

10.1016/j.physbeh.2011.07.029

Blaser RE, Vira DG. 2014. Experiments on learning in zebrafish (Danio rerio): a promising model of neurocognitive function. Neuroscience \& Biobehavioural Reviews, 42: 224-231. DOI: https://doi.org/10.1016/j.neubiorev.2014.03.003.

Cachat J, Canavello P, Elegante M, Bartels B, Hart P, Bergner C, Egan R, Duncan A, Tien D, Chung A, Wong K, Goodspeed J, Tan J, Grimes C, Elkhayat S, Suciu C, Rosenberg M, Chung KM, Kadri F, Roy S, Gaikwad S, Stewart A, Zapolsky I, Gilder T, Mohnot S, Beeson E, Amri H, Zukowska Z, Soignier RD, Kaleuff AV. 2010. Modeling withdrawal syndrome in zebrafish. Behavior Brain Research, 208: 371-376. DOI:

https://doi.org/10.1016/j.bbr.2009.12.004.

Collier AD, Echevarria DJ. 2013. The utility of the zebrafish model in conditioned place preference to assess the rewarding effects of drugs. Behaviour Pharmacology, 24: 375-383. DOI: https://doi.org/10.1097/FBP.0b013e328363d14a.

Davitz JR, Donald JM. 1955. Socially facilitated reduction of a fear response in rats. Journal of Comparative Psychology, 48: 149-151. DOI: https://doi.org/10.1037/h0046411.

Dean R, Duperrault E, Newton D, Krook J, Ingraham E, Gallup J, Franczak BC, Hamilton TJ. 2020. Opposing effects of acute and repeated nicotine exposure on boldness in zebrafish. Scientific Reports, 10: 8570. DOI: https://doi.org/10.1038/s41598-020-65382-6

Dresoti E, Lopes G, Kampff AR, Wilson WW. 2015. Development of social behavior in young zebrafish. Frontiers in Neural Circuits, 9:39. https://doi.org/10.3389/fncir.2015.00039

Echevarria DJ, Hammock CM, Pratt DW, Hosemann, JD. 2008. A novel behavioural test battery to assess global drug effects using the zebrafish. International Journal of Comparative Psychology, 21: 19-35.

Egan RJ, Bergner CL, Hart PC, Cachat JM, Canavello PR, Elegante MF, Elkhayat SI, Bartels BK, Tien AK, Tien DH, Mohnot S, Beeson E, Glasgow E, Amri H, Zukowska Z, Kalueff AV. 2009. Understanding behavioral and physiological phenotypes of stress and anxiety in zebrafish. Behavioural Brain Research, 205: 38-44. Doi: 10.1016/j.bbr.2009.06.022

Faustino AI, Tacão-Monteiro A, Oliveira RF. 2017. Mechanisms of social buffering of fear in zebrafish. Scientific Reports, 7: 44329. DOI: https://doi.org/10.1038/srep44329.

Fontana BD. Duarte T, Muller TE, Canzian J, Ziana PR, Mezzomo NJ, Parker MO, Rosemberg DB. 2020. Concomitant taurine exposure counteracts ethanol-induced changes in locomotor and 
505 anxiety-like responses in zebrafish. Psychopharmacology, 237: 735-743. DOI:

506 https://doi.org/10.1007/s00213-019-05410-0

507

508 Fox J. 2016. Applied Regression Analysis and Generalized Linear Models, Third Edition. Sage.

509 Fox J. and Weisberg, S. 2019. An R Companion to Applied Regression, Third Edition, Sage

510

511

512

513

514

515

516

517

518

519

520

521

522

523

524

525

526

527

528

529

530

531

532

533

534

535

536

537

538

539

540

541

542

543

544

545

546

547

Gebauer DL, Pagnussat N, Piato AL, Schaefer IC, Bonn CD, Lara DR. 2011. Effects of anxiolytics in zebrafish: Similarities and differences between benzodiazepines, buspirone and ethanol. Pharmacology Biochemistry and Behavior, 99: 480- 487. DOI: 10.1016/j.pbb.2011.04.021

Gerald R, Lee V, Blaser R. 2006. Effects of acute and chronic ethanol exposure on the behavior of adult zebrafish (Danio rerio). Pharmacology Biochemistry and Behavior, 85: 752-761. DOI: https://doi.org/10.1016/j.pbb.2006.11.010.

Gerlai R, Ahmad F, Prajapati S. 2008. Differences in acute alcohol-induced behavioral responses among zebrafish populations. Alcoholism, Clinical and Experimental Research, 32: 1763-177s. DOI: $10.1111 / j .1530-0277.2008 .00761 . x$

Hamilton TJ, Myggland A, Duperreault E, May Z, Gallup J, Powell RA, Schalomon M, Digweed SM. 2016. Episodic-like memory in zebrafish. Animal Cognition. 19(6):1071-1079. doi: 10.1007/s 10071-016-1014-1.

Hamilton TJ, Morrill A, Lucas K, Gallup J, Harris M, Healey M, Pitman T, Schalomon M, Digweed S, Tresguerres M. 2017. Establishing zebrafish as a model to study the anxiolytic effects of scopolamine. Scientific Reports, 7: 1-9. DOI: https://doi.org/10.1038/s41598-01715374-w.

Holcombe A, Howorko A, Powell RA, Schalomon M, Hamilton TJ. 2013. Reversed scototaxis during withdrawal after daily-moderate, but not weekly-binge, administration of ethanol in zebrafish. PloS one, 8. DOI: https://doi.org/10.1371/journal.pone.0063319.

Hostinar CE, Johnson AE, Gunnar MR. 2015. Early social deprivation and the social buffering of cortisol stress responses in late childhood: An experimental study. Developmental Psychology, 51: 1597-1608. DOI: https://doi.org/10.1037/dev0000029.

Johnson A, Hamilton TJ. 2017. Modafinil decreases anxiety-like behaviour in zebrafish. PeerJ, 5. DOI: https://doi.org/10.7717/peerj.2994.

Kikusui T, Winslow JT, Mori Y. 2006. Social buffering: relief from stress and anxiety. Philosophical Transactions: Biological Sciences, 1476: 2215-2228. DOI: http://doi.org/10.1098/rstb.2006.1941.

Peer) reviewing PDF | (2020:06:49781:3:0:NEW 19 Nov 2020) 
548 Krook JT, Duperreault E, Newton D, Ross MS, Hamilton TJ. 2019. Repeated ethanol exposure 549 increases anxiety-like behaviour in zebrafish during withdrawal. PeerJ, 7. DOI:

550 https://doi.org/10.7717/peerj.6551.

551

552

553

554

555

556

557

558

559

560

561

562

563

564

565

566

567

568

569

570

571

572

573

574

575

576

577

578

579

580

581

582

583

584

585

586

587

588

589

590

591

592

593

Latané B. Gregariousness and fear in laboratory rats. 1969. Journal of Experimental Social Psychology, 5: 61-69. DOI: https://doi.org/10.1016/0022-1031(69)90006-7.

Leighton PLA, Nadolski NJ, Morrill A, Hamilton TJ, Allison WT. 2018. An ancient conserved role for prion protein in learning and memory. Biology Open, 7. DOI:

https://doi.org/10.1242/bio.025734.

Liddell H. 1949. Some specific factors that modify tolerance for environmental stress. Research publications-Association for Research in Nervous and Mental Disease, 29: 155-171.

Masserman JH. 1943. Behavior and neurosis: An experimental psychoanalytic approach to psychobiologic principles. Chicago, IL: University of Chicago Press.

Maximino C, de Brito TM, da Silva Batista AW, Herculano AM, Morato S, Gouveia Jr A. 2010. Measuring anxiety in zebrafish: a critical review. Behavior Brain Research, 214: 157-171. DOI: https://doi.org/10.1016/j.bbr.2010.05.031.

May Z, Morrill A, Holcombe A, Johnston T, Gallup J, Fouad K, Schalomon M, Hamilton TJ. 2016. Object recognition memory in zebrafish. Behavior Brain Research, 296: 199-210. DOI: https://doi.org/10.1016/j.bbr.2015.09.016.

Munzel U, Brunner E. 2000. Nonparametric methods in multivariate factorial planning. Journal of Statistical Planning and Inference, 88(1): 117-132. DOI: https://doi.org/10.1016/S0378$\underline{3758(99) 00212-8}$

Ou M, Hamilton TJ, Eom J, Lyall EM, Gallup J, Jiang A, Lee J, Close DA, Yun S-S, Brauner CJ. 2015. Responses of pink salmon to $\mathrm{CO}_{2}$-induced aquatic acidification. Nature Climate Change. 5(10):950-955. doi: 10.1038/nclimate2694.

Oliveira RF, Faustino AI. 2017. Social information use in threat perception: Social buffering, contagion and facilitation of alarm responses. Communicative \& Integrative Biology, 10: 44329. DOI: https://doi.org/10.1080/19420889.2017.1325049.

Rubinstein DI. 1978. On predation, competition, and the advantages of group living.

Social Behavior, 205-231. DOI: https://doi.org/10.1007/978-1-4684-2901-5_9.

Sison M, Gerlai R. 2010. Associative learning in zebrafish (Danio rerio) in the plus maze. Behavior Brain Research, 207: 99-104. DOI: https://doi.org/10.1016/j.bbr.2009.09.043.

Speedie N, Gerlai R. 2008. Alarm substance induced behavioral responses in zebrafish (Danio rerio). Behavior Brain Research,188: 168-177. DOI: https://doi.org/10.1016/j.bbr.2007.10.031.

Peer) reviewing PDF | (2020:06:49781:3:0:NEW 19 Nov 2020) 
594 Stewart A, Gaikwad S, Kyzar E, Green J, Roth A, Kalueff AV. 2012. Modeling anxiety using 595 adult zebrafish: a conceptual review. Neuropharmacology, 62: 135-143. DOI:

596 https://doi.org/10.1016/j.neuropharm.2011.07.037.

597

598 Suboski MD, Bain S, Carty AE, McQuoid LM, Seelen MI, Seifert M. 1990. Alarm reaction in 599 acquisition and social transmission of simulated predator recognition by zebra danio fish

600 (Brachydanio rerio). Journal of Comparative Psychology, 104: 101-112. DOI: 10.1037/07356017036.104 .1 .101

602

603 Thomson HR, Lamb SD, Besson AA, Johnson SL. 2020. Long-term repeatability of behaviours 604 in zebrafish (Danio rerio). Ethology, 126: 803-811. DOI: 10.1111/eth.13038 


\section{Figure 1}

Experimental dosing set-up

(A) Isolated and (B) In-view dosing. An Individual fish was netted from the holding tank and placed into one of the two dosing containers. In-view fish had visual access to 12 conspecifics held in the conspecific tank but were not able to see the other fish being dosed. Fish remained in the dosing containers for 30-minutes prior to behavioural testing. C) The circular arena used was $34 \mathrm{~cm}$ in diameter and $16 \mathrm{~cm}$ in height. D) The novel object used was a multi-coloured LEGO figurine. E) The thigmotaxis, transition and inner zones were calibrated to 34,23 and $12 \mathrm{~cm}$ in diameter respectively. 
A

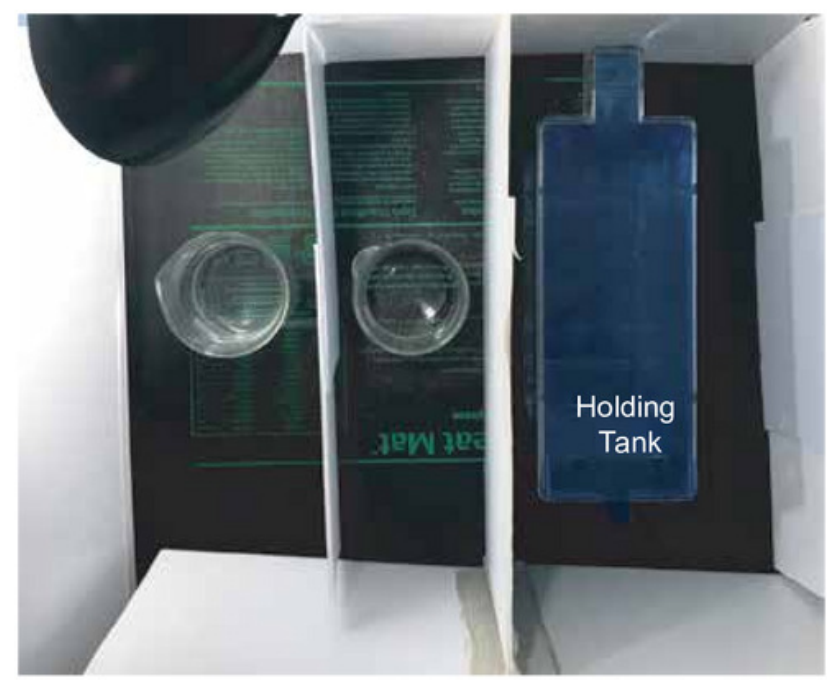

C

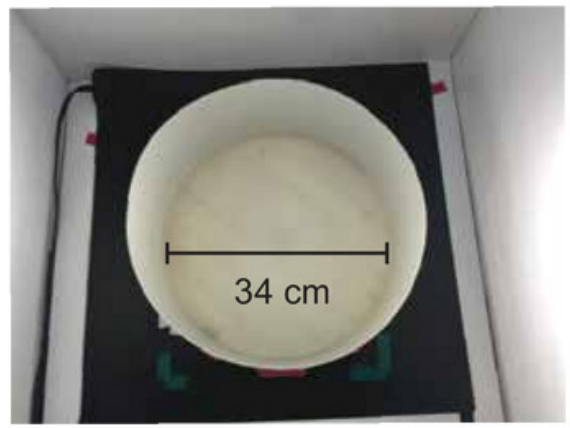

D

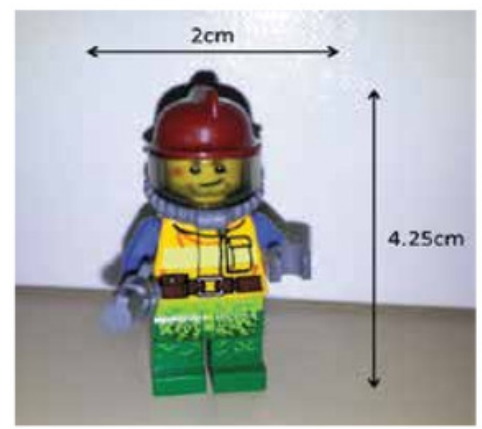

B
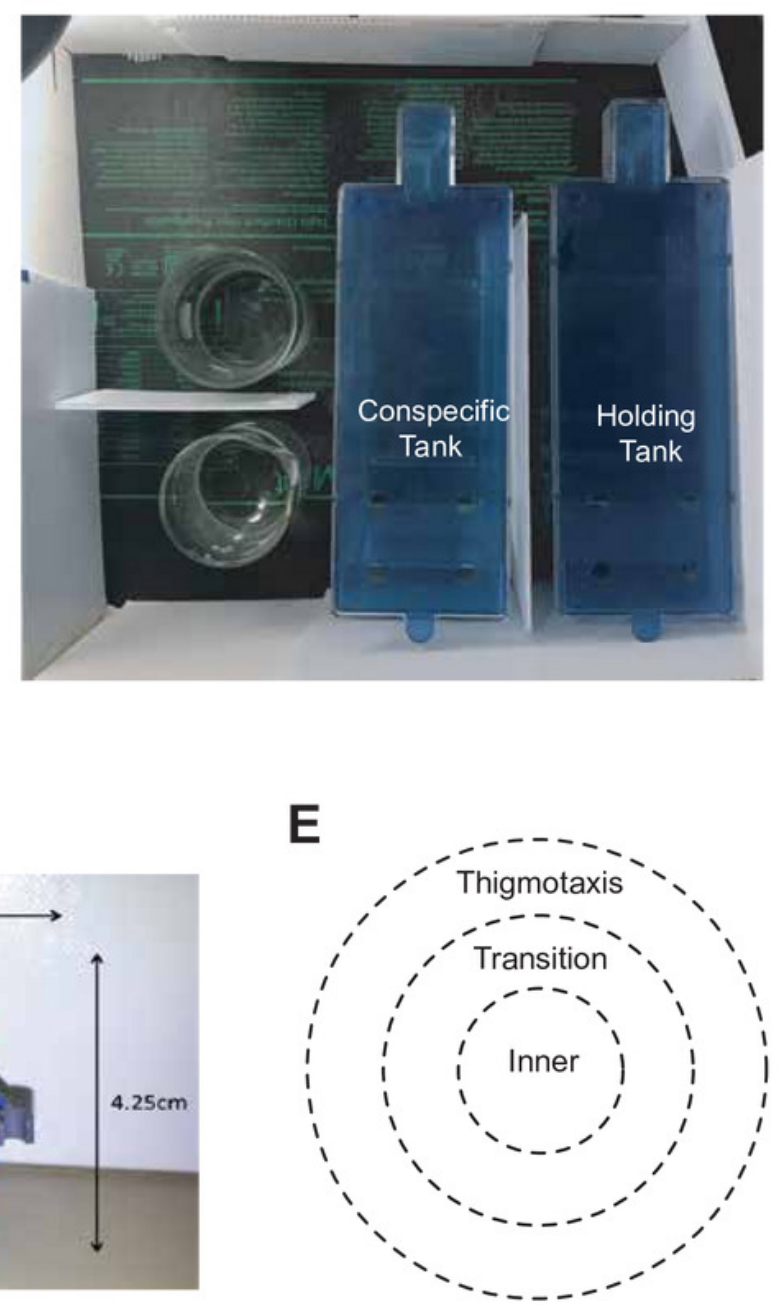
Figure 2

Experimental set up for tracking behaviour during testing

(A) Isolated dosing, or (B) In-view dosing. The circle in the bottom left of figure (B) represents the virtual zones created in Ethovision to test whether fish spend more time on the side of the beaker closest to conspecifics when in view. 
A

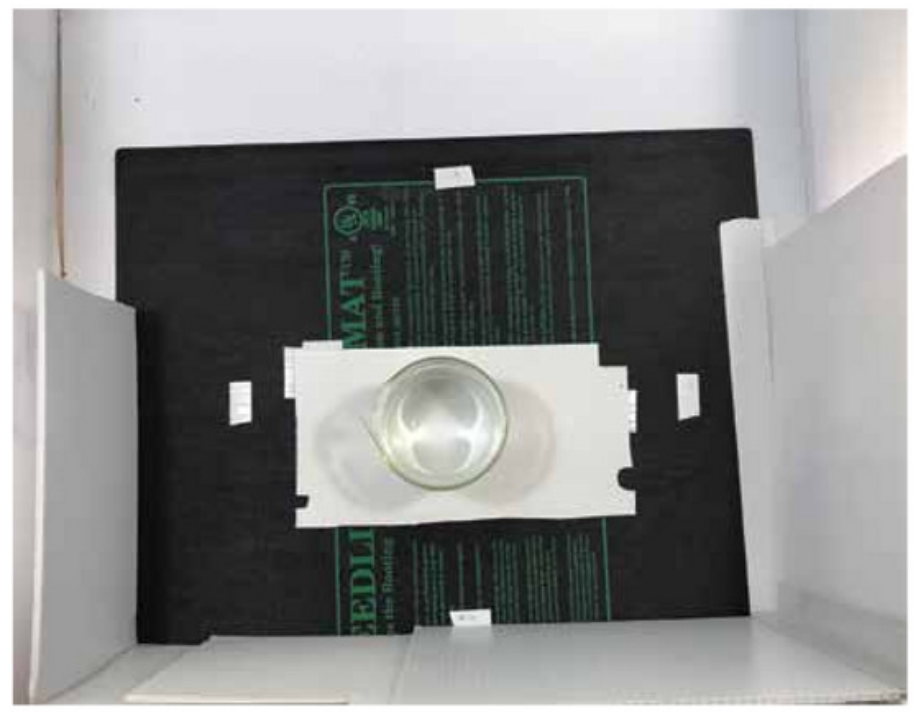

B

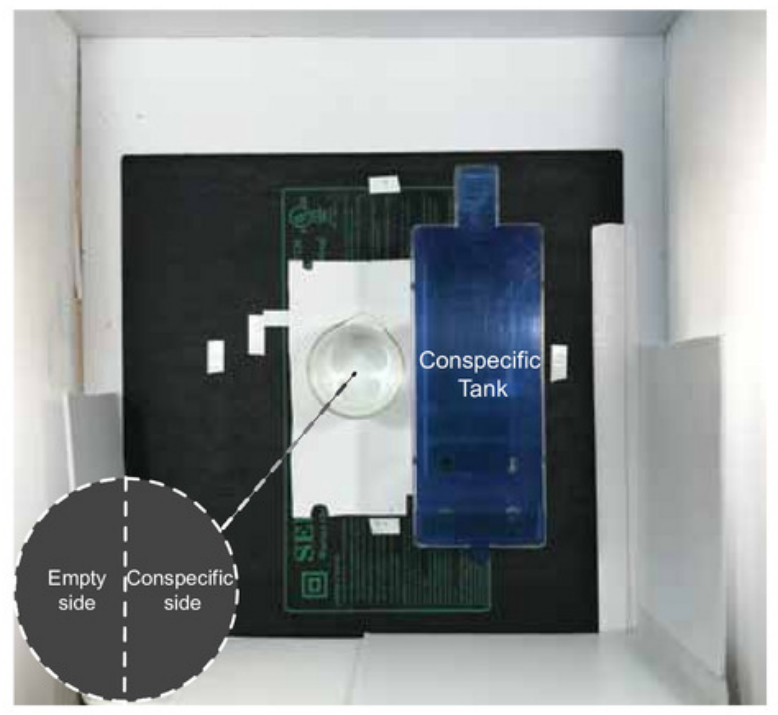




\section{Figure 3}

Effects of social context and ethanol on zone preference

The time, in seconds, fish spent in the thigmotaxis (A), transition (B) and inner zones (C). (D) and (E) represent the effect of social context and substance on the distance fish moved (D) and the time fish spent immobile (E). Individual data points represent mean values ( $n=15$ per group). Error bars represent the SE and * identifies significant differences between group means using $95 \%$ C.I. $* * P<0.01 ; * * * P<0.001 ; * * * P<0.0001$ 
A

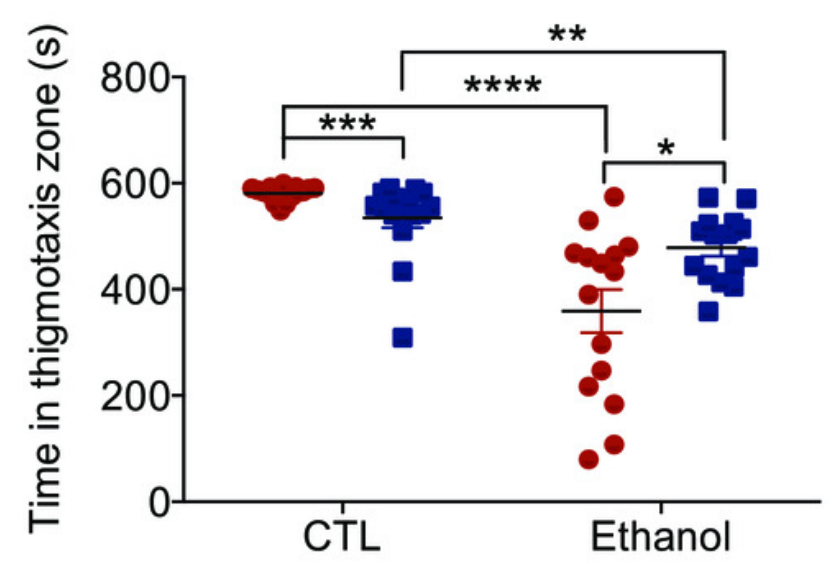

C

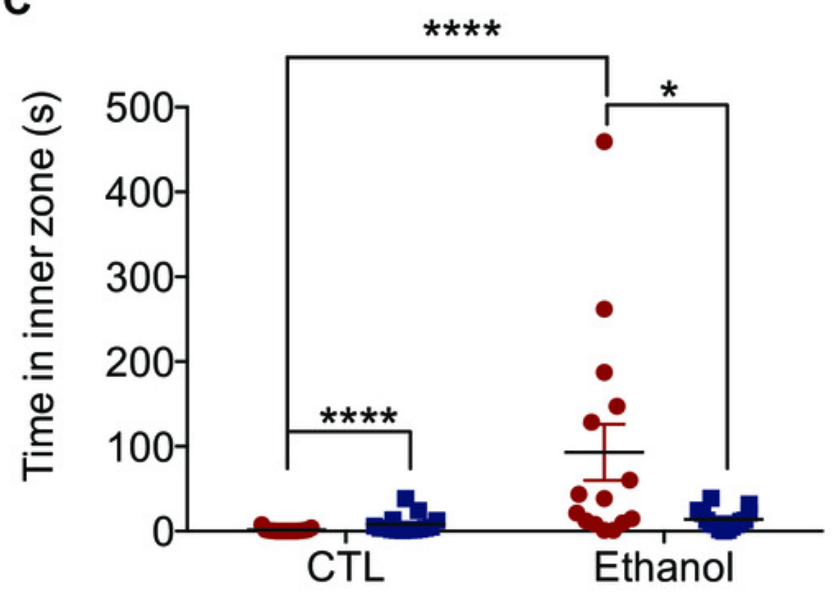

E

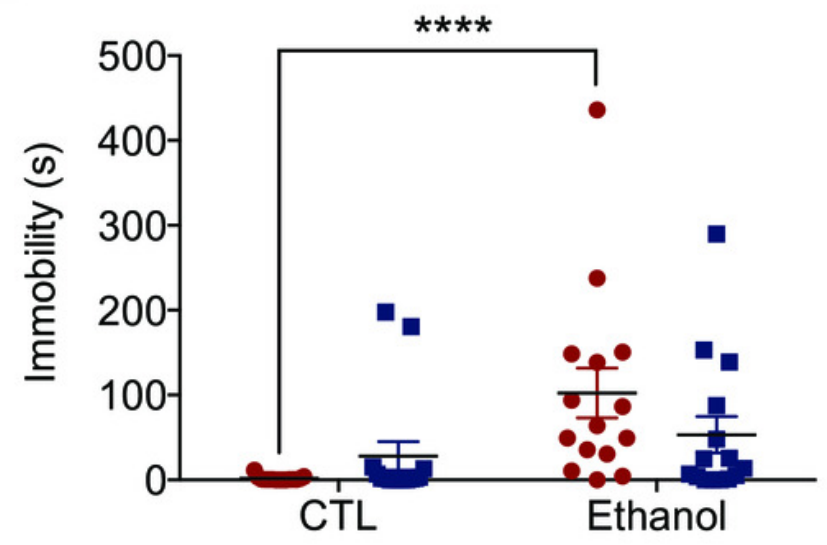

B

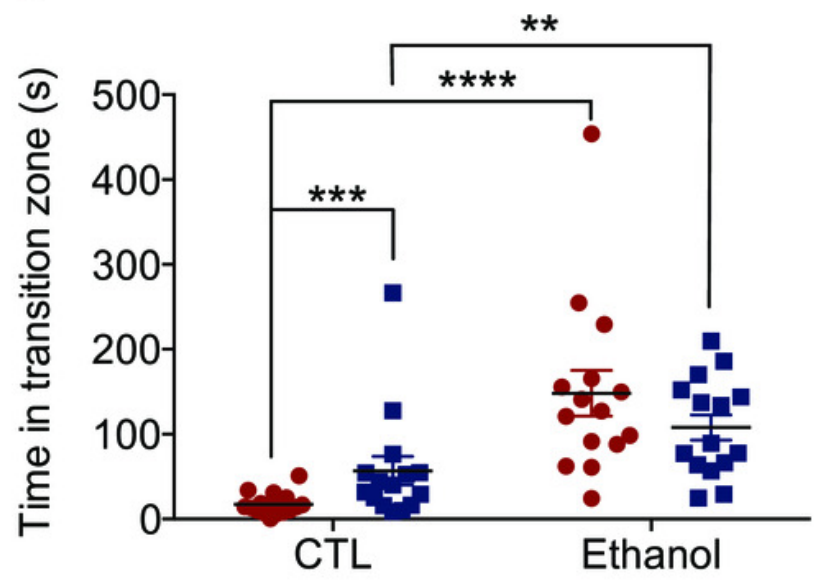

D

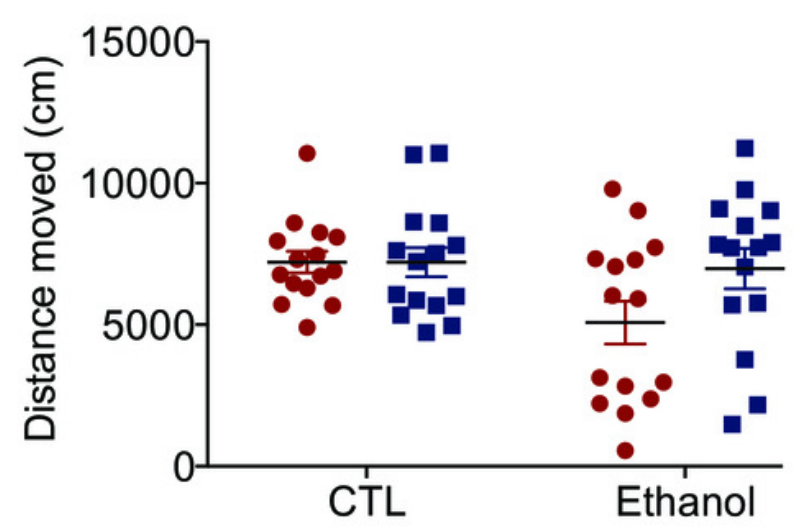




\section{Figure 4}

Distance and side preferences during the dosing procedure

(A) The distance, Isolated and In-view-CTL fish moved and the (B) amount of time, in seconds, In-view-CTLs spent on either side of the dosing container during dosing. The social condition did not have a significant effect on the distance fish moved, however when in-view, fish had a significant preference for the side of the dosing container closest to conspecifics. Data was analyzed using independent $t$-tests. Individual data points ( $n=15$ per group) represent mean values \pm SEM. Error bars represent SE and * identifies significant differences between group means using $95 \%$ C.I. ${ }^{* * * *} P<0.0001$. 
A

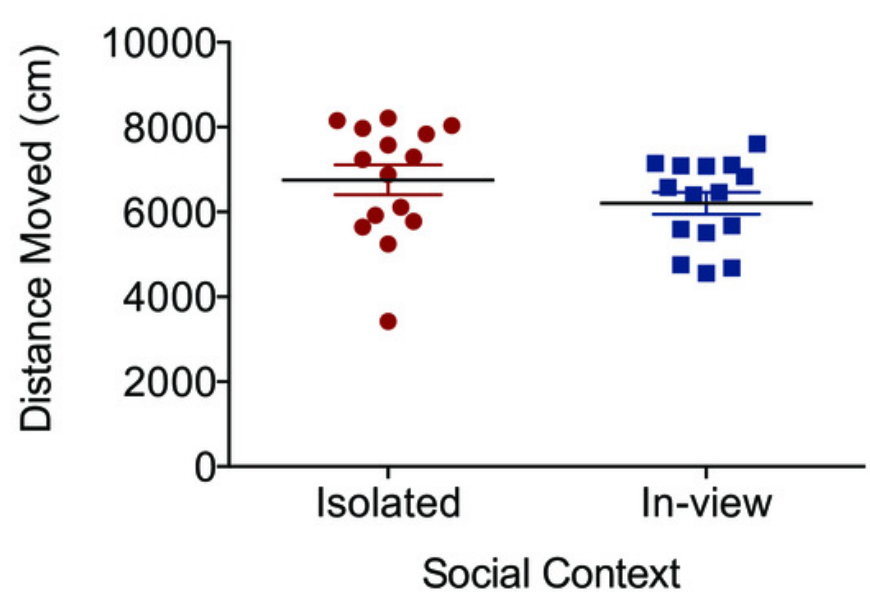

B

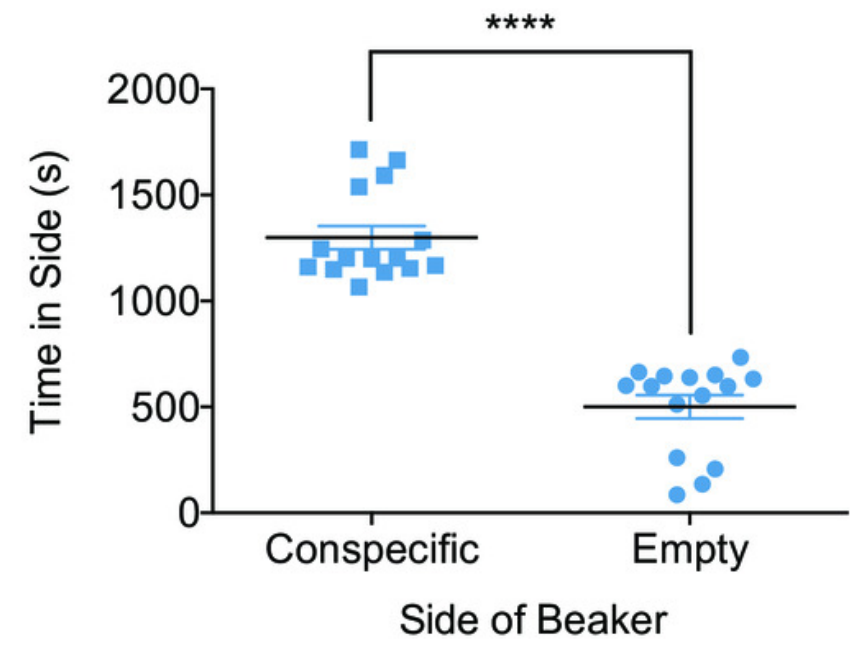

